\title{
GENERALIZED QUASILINEARIZATION METHOD FOR NONLINEAR FUNCTIONAL DIFFERENTIAL EQUATIONS
}

\author{
BASHIR AHMAD and REHMAT ALI KHAN \\ Quaid-i-Azam University, Department of Mathematics, \\ Islamabad-Pakistan \\ and \\ S. SIVASUNDARAM \\ Embry-Riddle Aeronautical University, Department of Mathematics, \\ Daytona Beach, FL 32114, USA
}

(Received March, 2002; Revised October, 2002)

\begin{abstract}
We develop a generalized quasilinearization method for nonlinear initial value problems involving functional differential equations and obtain a sequence of approximate solutions converging monotonically and quadratically to the solution of the problem. In addition, we obtain a monotone sequence of approximate solutions converging uniformly to the solution of the problem, possessing the rate of convergence higher than quadratic.

Key words: Generalized Quasilinearization, Functional Differential Equations, Quadratic convergence.
\end{abstract}

AMS (MOS) subject classification: 34A45, 34B15

\section{Introduction}

The method of quasilinearization pioneered by Bellman and Kalaba [1] provides a descent approach for obtaining approximate solutions to a nonlinear differential equation provided the nonlinearity involved is convex. Recently, this method has been generalized by relaxing the convexity assumption. This development was so significant that it received much attention and the generalized quasilinearization method was applied to a variety of problems $[2,3,4,5,6,7,9,10,11,12]$. For a complete survey of the generalized quasilinearization technique, see [8].

The future state of a physical system depends not only on the present state but also on its past history. Functional differential equations provide a mathematical model for such physical systems in which the rate of change of the system may not depend on the influence of its hereditary effects. The impetus has mainly been due to developments in control theory, mathematical biology, mathematical economics, and the theory of systems which communicate through less channels. The simplest type of such a system is a differential-difference equation of the form $x^{\prime}(t)=f(t, x(t), x(t-\tau))$, where $\tau>0$ 
is a positive constant. More general systems may be described by $x^{\prime}(t)=f\left(t, x_{t}\right)$ where $f$ is a suitable functional.

The aim of this paper is to consider a nonlinear initial value problem (IVP)involving functional differential equation and develop a method of quasilinearization for this problem without requiring the function involved to be convex/concave. A monotone sequence of approximate solutions converging monotonically to a solution of the problem, with convergence higher than quadratic $(k \geq 2)$ is obtained.

\section{Preliminaries}

Given any $\tau>0$, let $\Gamma=C[[-\tau, 0], R]$ and for any $\phi \in \Gamma$, define the norm

$$
\|\phi\|_{o}=\max _{-\tau \leq s \leq 0}|\phi| \text {. }
$$

For any $t \geq 0$, let $x_{t}$ denotes a translation of the restriction of $x \in C[[-\tau, T], R]$ to the interval $[-\tau, 0]$ and it is defined by

$$
x_{t}(s)=x(s+t), \quad-\tau \leq s \leq 0, t \in J=[-\tau, T] .
$$

Now, consider the IVP for the functional differential equation

$$
x^{\prime}=f\left(t, x_{t}\right), x_{o}=\phi_{o} \in \digamma, t \in[0, T]=J,
$$

where $f \in C[J \times \Gamma, R]$, and let $\bar{J}=[-\tau, T]$. A function $\alpha \in C[[-\tau, T], R]$ is a lower solution of (2.1) if

$$
D_{+} \alpha(t) \leq f\left(t, \alpha_{t}\right), t \in[0, T], \alpha_{0} \leq \phi_{o},
$$

and $\beta \in C[[-\tau, T], R]$ is an upper solution of (2.1) if

$$
D_{+} \beta(t) \geq f\left(t, \beta_{t}\right), t \in[0, T], \beta_{0} \geq \phi_{o} .
$$

Now, we state the following results, which play an important role in the sequel (for the proof, see p: 34-35, [4]).

Theorem 2.1: $\quad$ Let

(1) $f \in C[J \times \Gamma, R]$ and $f(t, x, \phi)$ be quasinondecreasing in $\phi$ for each $t \in J$ and satisfy the condition

$$
f(t, \phi)-f(t, \psi) \leq L(\phi-\psi), t \in J,
$$

where $\phi, \psi \in \Gamma$, such that $\phi(s) \leq \psi(s),-\tau \leq s \leq 0$, and $0<L<1$.

(2) $\alpha, \beta \in C[[-\tau, T], R]$ be such that

$$
\begin{aligned}
& D_{+} \alpha(t) \leq f\left(t, \alpha_{t}\right) \\
& D_{+} \beta(t) \geq f\left(t, \beta_{t}\right) \quad \text { for } t \in[0, T],
\end{aligned}
$$

and $\alpha_{o}(s) \leq \beta_{o}(s),-\tau \leq s \leq 0$.

Then,

$$
\alpha(t) \leq \beta(t), \text { for } t \in \bar{J} .
$$


Theorem 2.2: $\quad$ Let $\alpha(t), \beta(t)$ be lower and upper solutions of (2.1). Let $f \in C[J \times$ $\Gamma, R]$ and $f(t, \phi)$ be quasinondecreasing in $\phi$ for each $t \in J$. Suppose that $x=x\left(0, \phi_{o}\right)$ is any solution of $(2.1)$ defined on $[0, T]$. Then,

$$
\alpha(t) \leq x\left(0, \phi_{o}\right)(t) \leq \beta(t) .
$$

Theorem 2.3: Let $f \in C[J \times R \times \Gamma, R]$ and $f(t, x, \phi)$ be nondecreasing in $\phi$ for each $(t, x)$. Let $x, y \in C[[-\tau, T], R]$ and $x_{o} \leq y_{o}$. Assume further that $x^{\prime}(t) \leq f\left(t, x(t), x_{t}\right)$, $y^{\prime}(t) \geq f\left(t, y(t), y_{t}\right)$. Then $x(t) \leq y(t)$, for $t \in J=[-\tau, T]$.

Theorem 2.4: Let $y(u), z(u)$ be lower and upper solutions of $(2.1)$ and $f(t, x, \phi) \in$ $C[J \times R \times \Gamma, R]$ be nondecreasing in $\phi$ for each $(t, x)$. Suppose that $x=x\left(0, \phi_{o}\right)$ is any solution of (2.1) defined on $[0, T]$, such that

$$
y_{o} \leq \phi_{o} \leq z_{o}
$$

Then,

$$
y(t) \leq x\left(0, \phi_{o}\right)(t) \leq z(t)
$$

\section{Main Result}

Theorem 3.1: Assume that

$\left(A_{1}\right) f \in C[J \times \Gamma, R]$ and $f(t, \phi)$ is quasinondecreasing in $\phi$ for each $t \in J$.

$\left(A_{2}\right) \alpha, \beta \in C[\bar{J}, R] \cap C^{1}[J, R]$ are lower and upper solutions of (1) satisfying

$$
\alpha(t) \leq \beta(t), \quad t \in \bar{J} .
$$

$\left(A_{3}\right)$ The derivatives $f_{\phi}(t, \phi)$ and $f_{\phi \phi}(t, \phi)$ exist and are continuous and satisfying, $f_{\phi \phi}(t, \phi) \geq-2 m, 0 \leq f_{\phi}(t, \phi) \leq L$, for some $m>0,1>L>0, t \in J$.

Then there exists a monotone sequence $\left\{u_{n}(t)\right\}$, which converges uniformly to the unique solution of (2.1) on $J$ and that the convergence is quadratic.

Proof: In view of the assumption $\left(A_{3}\right)$, we can write

$$
f(t, \phi) \geq f(t, \psi)+\left(f_{\phi}(t, \psi)+2 m \psi\right)(\phi-\psi)-m\left(\phi^{2}-\psi^{2}\right),
$$

where,

$$
\alpha_{t} \leq \psi \leq \phi \leq \beta_{t} \quad \text { for } t \in J, \quad \text { and } \phi, \psi \in \Gamma .
$$

Define the functional $F(t, \phi, \psi)$ as

$$
F(t, \phi, \psi)=f(t, \psi)+\left(f_{\phi}(t, \psi)+2 m \psi\right)(\phi-\psi)-m\left(\phi^{2}-\psi^{2}\right) .
$$

We observe that

$$
F(t, \phi, \psi) \leq f(t, \phi) \quad \text { and } \quad F(t, \phi, \phi)=f(t, \phi) .
$$


Setting $\alpha=u_{o}$, and consider the IVP for functional differential equation:

$$
u^{\prime}=F\left(t, u_{t}, u_{o, t}\right), \quad u_{o}=\phi_{o} \in \Gamma, t \in J .
$$

Observe that

$$
\begin{aligned}
\frac{\partial}{\partial \phi} F\left(t, u_{t}, \phi\right) & =\frac{\partial}{\partial \phi}\left[f(t, \phi)+\left(f_{\phi}(t, \phi)+2 m \phi\right)\left(u_{t}-\phi\right)-m\left(u_{t}^{2}-\phi^{2}\right)\right] \\
& =\left(f_{\phi \phi}(t, \phi)+2 m\right)\left(u_{t}-\phi\right) \geq 0
\end{aligned}
$$

and

$$
u_{o, t} \leq \phi \leq u_{t} \leq \beta_{t} \quad \text { for } t \in J \text {, and } \phi, u_{t} \in \Gamma \text {, }
$$

which implies that $F\left(t, u_{t}, \phi\right)$ is nondecreasing in $\phi$ for each $\left(t, u_{t}\right)$. Furthermore, in view of (3.3) and (3.4), we have

$$
\begin{aligned}
F\left(t, \phi_{1}, u_{o, t}\right)-F\left(t, \phi_{2}, u_{o, t}\right) & =\left(f_{\phi}\left(t, u_{o, t}\right)+2 m u_{o, t}\right)\left(\phi_{1}-\phi_{2}\right)-m\left(\phi_{1}^{2}-\phi_{2}^{2}\right) \\
& =\left(f_{\phi}\left(t, u_{o, t}\right)+2 m u_{o, t}-m\left(\phi_{1}+\phi_{2}\right)\left(\phi_{1}-\phi_{2}\right)\right. \\
& =\left(f_{\phi}\left(t, u_{o, t}\right)-m\left[\left(\phi_{1}-u_{o, t}\right)+\left(\phi_{2}-u_{o, t}\right)\right]\right)\left(\phi_{1}-\phi_{2}\right) \\
& \leq f_{\phi}\left(t, u_{o, t}\right)\left(\phi_{1}-\phi_{2}\right) \\
& \leq L\left(\phi_{1}-\phi_{2}\right), \text { for some } 1>L>0,
\end{aligned}
$$

where $u_{o, t} \leq \phi_{2} \leq \phi_{1} \leq \beta_{t}$ for $t \in J$, and $\phi_{1}, \phi_{2} \in \Gamma$. This implies that $F(t, \phi, \psi)$ satisfies one-sided Lipschitz condition. Since $F\left(t, u_{t}, u_{o, t}\right)$ is quasimonotone nondecreasing and satisfies one-sided Lipschitz condition, it follows that (3.5) has a unique solution $u_{1}(t)$, with $u_{1,0}=\phi_{o}$ on $J$. Now, in view of $\left(\mathrm{A}_{2}\right)$ and (3.4), we have

$$
D_{+} u_{o}(t) \leq f\left(t, u_{o, t}\right)=F\left(t, u_{o, t}, u_{o, t}\right), \quad u_{o, 0} \leq \phi_{o}
$$

and

$$
D_{+} \beta(t) \geq f\left(t, \beta_{t}\right) \geq F\left(t, \beta_{t}, u_{o, t}\right), \quad \beta_{0} \geq \phi_{o} .
$$

It follows that $u_{o}(t), \beta(t)$ are lower and upper solutions of (3.5). Also,

$$
u_{o, 0} \leq u_{1,0} \leq \beta_{0}
$$

Thus, by Theorem 2.2, we conclude that

$$
u_{o, t} \leq u_{1, t} \leq \beta_{t} \text { for } t \in J .
$$

Now, consider the IVP for the functional differential equation

$$
u^{\prime}=F\left(t, u_{t}, u_{1, t}\right), \quad u_{0}=\phi_{o}=u_{1,0} \quad, t \in J .
$$

Repeating the procedure used earlier, (3.7) has a unique solution $u_{2}(t)$, with

$$
u_{2,0}=\phi_{o} .
$$

In view of (3.4), the quasinondecreasing nature of $F(t, \phi, \psi)$, and the fact that $u_{1}(t)$ is a solution of (3.5), we obtain

$$
D_{+} u_{1}(t)=F\left(t, u_{1, t}, u_{o, t}\right) \leq F\left(t, u_{1, t}, u_{1, t}\right), \quad u_{1,0}=\phi_{o},
$$


and,

$$
D_{+} \beta(t) \geq f\left(t, \beta_{t}\right) \geq F\left(t, \beta_{t}, u_{1, t}\right), \quad \beta_{0} \geq \phi_{o} .
$$

It follows that $u_{1}(t), \beta(t)$ are lower and upper solutions of (3.7), and since

$$
u_{1,0} \leq u_{2,0} \leq \beta_{0}
$$

by Theorem 2.2 , we have that

$$
u_{1, t} \leq u_{2, t} \leq \beta_{t} \quad \text { for } \quad t \in J .
$$

Continuing in the same way, we obtain a monotone sequence $\left\{u_{n, t}\right\}$ satisfying

$$
u_{o, t} \leq u_{1, t} \leq u_{2, t} \leq \ldots \leq u_{n, t} \leq \beta_{t} \quad \text { for } \quad t \in J,
$$

where the element $u_{n, t}$ of the sequence is a solution of the IVP

$$
u^{\prime}(t)=F\left(t, u_{t}, u_{n-1, t}\right), \quad u_{0}=\phi_{o}=u_{n, 0} \quad, t \in J .
$$

Since the sequence $\left\{u_{n, t}\right\}$ is monotone, it follows that it has a pointwise limit $x_{t}$. To show that $x_{t}$ is in fact a solution of (2.1) we notice that $u_{n, t}$ is a solution of the following linear IVP for functional differential equation:

$$
\begin{aligned}
u^{\prime}(t) & =F\left(t, u_{n, t}, u_{n-1, t}\right), \quad u_{n, 0}=\phi_{o}, t \in J \\
& =\sigma_{n, t} .
\end{aligned}
$$

where, $\sigma_{n, t}=F\left(t, u_{n, t}, u_{n-1, t}\right), t \in J$. Since $F$ is continuous on $[0, T]$, it follows that $\left\{\sigma_{n, t}\right\}$ is bounded on $[0, T]$. Also,

$$
\text { Limit }_{n \rightarrow \infty} \sigma_{n, t}=F\left(t, x_{t}, x_{t}\right)=f\left(t, x_{t}\right), t \in J .
$$

Thus, from (3.11), we have

$$
u_{n, t}=\int_{0}^{t} \sigma_{n, s} d s
$$

Taking limit $n \rightarrow \infty$,we obtain

$$
x_{t}=\int_{0}^{t} f\left(s, x_{t}\right) d s,
$$

which is a solution of (2.1). Finally, we have to show that the convergence is quadratic. For that, we define

$$
e_{n}(t)=x(t)-u_{n}(t), t \in \bar{J} .
$$

Observe that $e_{n}(t) \geq 0$ and $e_{n}(s)=x(s)-u_{n}(s)=x_{0}-u_{n, 0}=\phi_{o}-\phi_{o}=0$. Now,

$$
\begin{aligned}
e_{n}^{\prime}(t)= & x^{\prime}(t)-u_{n}^{\prime}(t) \\
= & f\left(t, x_{t}\right)-F\left(t, u_{n, t}, u_{n-1, t}\right) \\
= & f\left(t, x_{t}\right)-\left[f\left(t, u_{n-1, t}\right)+\left(f_{\phi}\left(t, u_{n-1, t}\right)+2 m u_{n-1, t}\right)\left(u_{n, t}-u_{n-1, t}\right)\right. \\
& \left.-m\left(u_{n, t}^{2}-u_{n-1, t}^{2}\right)\right] \\
= & f\left(t, x_{t}\right)-f\left(t, u_{n-1, t}\right)-\left(f_{\phi}\left(t, u_{n-1, t}\right)+2 m u_{n-1, t}\right)\left(u_{n, t}-u_{n-1, t}\right) \\
& \left.+m\left(u_{n, t}^{2}-u_{n-1, t}^{2}\right)\right] .
\end{aligned}
$$


Define

$$
G\left(t, x_{t}\right)=f\left(t, x_{t}\right)+m x_{t}^{2} .
$$

Notice that $G_{\phi \phi}\left(t, x_{t}\right)=f_{\phi \phi}\left(t, x_{t}\right)-2 m>0$, so that we can find $C>0$ such that

$$
0 \leq G_{\phi \phi}(t, \phi) \leq C \text {. }
$$

Using (3.16) in (3.15) yields

$$
\begin{aligned}
e_{n}^{\prime}(t)= & G\left(t, x_{t}\right)-G\left(t, u_{n-1, t}\right)-G_{\phi}\left(t, u_{n-1, t}\right)\left(u_{n, t}-u_{n-1, t}\right)-m\left(x_{t}^{2}-u_{n, t}^{2}\right) \\
= & \int_{0}^{1} G_{\phi}\left(t, s x_{t}+(1-s) u_{n-1, t}\right) e_{n-1, t} d s-G_{\phi}\left(t, u_{n-1, t}\right)\left[\left(x_{t}-u_{n-1, t}\right)\right. \\
& \left.-\left(x_{t}-u_{n, t}\right)\right]-m\left(x_{t}^{2}-u_{n, t}^{2}\right) \\
= & \int_{0}^{1} G_{\phi}\left(t, s x_{t}+(1-s) u_{n-1, t}\right) e_{n-1, t} d s-G_{\phi}\left(t, u_{n-1, t}\right) e_{n-1, t}+ \\
& {\left[G_{\phi}\left(t, u_{n-1, t}\right)-m\left(x_{t}+u_{n, t}\right)\right] e_{n, t} . } \\
\leq & \int_{0}^{1} G_{\phi}\left(t, s x_{t}+(1-s) u_{n-1, t}\right) e_{n-1, t} d s-G_{\phi}\left(t, u_{n-1, t}\right) e_{n-1, t} \\
& \left.+\left[G_{\phi}\left(t, u_{n-1, t}\right)-2 m u_{n-1, t}\right)\right] e_{n, t} \\
= & \int_{0}^{1} G_{\phi}\left(t, s x_{t}+(1-s) u_{n-1, t}\right) e_{n-1, t} d s-G_{\phi}\left(t, u_{n-1, t}\right) e_{n-1, t}+f_{\phi}\left(t, u_{n-1, t}\right) e_{n, t} \\
= & \int_{0}^{1}\left[G_{\phi}\left(t, s x_{t}+(1-s) u_{n-1, t}\right)-G_{\phi}\left(t, u_{n-1, t}\right)\right] e_{n-1, t} d s+f_{\phi}\left(t, u_{n-1, t}\right) e_{n, t} .
\end{aligned}
$$

Using $\left(\mathrm{A}_{3}\right)$ and $\left(\mathrm{A}_{4}\right)$, the last result becomes

$$
\begin{aligned}
e_{n}^{\prime}(t) & \leq \int_{0}^{1}\left[G_{\phi}\left(t, s x_{t}+(1-s) u_{n-1, t}\right)-G_{\phi}\left(t, u_{n-1, t}\right)\right] e_{n-1, t} d s+L \int_{-\tau}^{0} e_{n, t}(s) d s \\
& \leq \int_{0}^{1} L_{2}\left|s x_{t}+(1-s) u_{n-1, t}-u_{n-1, t}\right| e_{n-1, t} d s+L \int_{-\tau}^{0} e_{n, t}(s) d s \\
& \leq L_{2} e_{n-1, t}^{2}+L \int_{-\tau}^{0} e_{n, t}(s) d s=w^{\prime}(t) \text { (say). }
\end{aligned}
$$

Clearly, $w^{\prime}(t) \geq 0$. Since $e_{n}(t) \leq w(t)$ and $w(t)$ is nondecreasing in $t$, we get

$$
w(t) \leq L_{2} \int_{0}^{t} e_{n-1, s}^{2} d s+L \tau \int_{0}^{t} w(s) d s .
$$

Note that $w(0)=0$. By Gronwall's inequality [8], (3.18) can be written as

$$
\begin{aligned}
e_{n}(t) & \leq w(t) \leq L_{2} \int_{0}^{t} e^{L \tau(T-S)} e_{n-1, s}^{2} d s \\
& \leq L_{2} \frac{e^{L \tau T}}{L \tau} \max e_{n-1, t}^{2} \text { where } t \in J .
\end{aligned}
$$

Consequently,

$$
e_{n}(t) \leq L_{2} \frac{e^{L \tau T}}{L \tau} \max e_{n-1, t}^{2} \quad \text { where } t \in J
$$

This completes the proof.

Theorem 3.2: Assume that 
$\left(A_{1}\right) f \in C[J \times \Gamma, R]$ and $f(t, \phi)$ is quasinondecreasing in $\phi$ for each $t \in J$.

$\left(A_{2}\right) u_{o}, v_{o} \in C[\bar{J}, R] \cap C^{1}[J, R]$ are lower and upper solutions of (1) satisfying

$$
u_{o}(s) \leq v_{o}(s), \quad-\tau \leq s \leq 0 .
$$

$\left(A_{3}\right)$ The derivatives $\frac{\partial^{i}}{\partial \phi^{i}} f(t, \phi)(i=1,2,3, \ldots, k-1)$ exist and are continuous functions of $t$ on $[0, T]$, and $\frac{\partial^{k}}{\partial \phi^{k}} f(t, \phi)>-k ! m_{k}, m_{k}>0, t \in J$. Furthermore, $f_{\phi}(t, \phi) \leq$ $L \int_{-\tau}^{0} \psi(s) d s$, where $\phi, \psi \in \Gamma$ are such that

$$
u_{o, t} \leq \phi, \psi \leq v_{o, t} \quad \text { for } t \in J
$$

Then there exists a monotone sequence $\left\{u_{n}(t)\right\}$, which converges uniformly to the unique solution of (2.1) on $J$ and that the convergence is of order $k \geq 2$.

Proof: In view of assumption $\left(A_{3}\right)$ and generalized mean value theorem, we have

$$
f(t, \phi) \geq \sum_{i=0}^{k-1} \frac{\partial^{i}}{\partial \phi^{i}} f(t, \psi) \frac{(\phi-\psi)^{i}}{i !}-m_{k}(\phi-\psi)^{k},
$$

where

$$
u_{o, t} \leq \psi \leq \phi \leq v_{o, t} \quad \text { for } t \in J, \text { and } \phi, \psi \in \Gamma .
$$

Define the functional $F(t, \phi, \psi)$ as

$$
F(t, \phi, \psi)=\sum_{i=0}^{k-1} \frac{\partial^{i}}{\partial \phi^{i}} f(t, \psi) \frac{(\phi-\psi)^{i}}{i !}-m_{k}(\phi-\psi)^{k} .
$$

Observe that

$$
F(t, \phi, \psi) \leq f(t, \phi) \text { and } F(t, \phi, \phi)=f(t, \phi),
$$

and $F(t, \phi, \psi)$ is nondecreasing in $\psi$ for each $(t, \phi)$. Furthermore,

$$
\begin{aligned}
& F\left(t, \phi_{1}, \psi\right)-F\left(t, \phi_{2}, \psi\right) \\
= & \sum_{i=0}^{k-1} \frac{\partial^{i}}{\partial \phi^{i}} f(t, \psi) \frac{\left(\phi_{1}-\psi\right)^{i}}{i !}-m_{k}\left(\phi_{1}-\psi\right)^{k} \\
& -\sum_{i=0}^{k-1} \frac{\partial^{i}}{\partial \phi^{i}} f(t, \psi) \frac{\left(\phi_{2}-\psi\right)^{i}}{i !}+m_{k}\left(\phi_{2}-\psi\right)^{k} \\
= & {\left[\sum_{i=1}^{k-1} \frac{\partial^{i}}{\partial \phi^{i}} f(t, \psi) \frac{1}{i !} \sum_{j=0}^{i-1}\left(\phi_{1}-\psi\right)^{i-1-j}\left(\phi_{2}-\psi\right)^{j}\right]\left(\phi_{1}-\phi_{2}\right) } \\
& -m_{k} \sum_{j=0}^{k-1}\left(\phi_{1}-\psi\right)^{k-1-j}\left(\phi_{2}-\psi\right)^{j}\left(\phi_{1}-\phi\right) . \\
= & {\left[\sum_{i=1}^{k-1} \frac{\partial^{i}}{\partial \phi^{i}} f(t, \psi) \frac{1}{i !} \sum_{j=0}^{i-1}\left(\phi_{1}-\psi\right)^{i-1-j}\left(\phi_{2}-\psi\right)^{j}\right.}
\end{aligned}
$$




$$
\begin{aligned}
& \left.-m_{k} \sum_{j=0}^{k-1}\left(\phi_{1}-\psi\right)^{k-1-j}\left(\phi_{2}-\psi\right)^{j}\right]\left(\phi_{1}-\phi_{2}\right) . \\
\leq & \sum_{i=1}^{k-1} \frac{\partial^{i}}{\partial \phi^{i}} f(t, \psi) \frac{1}{i !} \sum_{j=0}^{i-1}\left(\phi_{1}-\psi\right)^{i-1-j}\left(\phi_{2}-\psi\right)^{j}\left(\phi_{1}-\phi_{2}\right) \leq M\left(\phi_{1}-\phi_{2}\right) .
\end{aligned}
$$

where

$$
0<\sum_{i=1}^{k-1} \frac{\partial^{i}}{\partial \phi^{i}} f(t, \psi) \frac{1}{i !} \sum_{j=0}^{i-1}\left(\phi_{1}-\psi\right)^{i-1-j}\left(\phi_{2}-\psi\right)^{j} \leq M .
$$

Clearly, $F(t, \phi, \psi)$ satisfies one-sided Lipschitz condition with respect to $\phi$ for each $(t, \psi)$.

Now, consider the IVP for the functional differential equation

$$
u^{\prime}(t)=F\left(t, u(t), u_{0, t}\right), \quad u_{0}=\phi_{o}, t \in J .
$$

Since $F\left(t, u(t), u_{0, t}\right)$ is quasimonotone nondecreasing and satisfies one-sided Lipschitz condition, it follows that (19) has a unique solution $u_{1}(t)$, with $u_{1,0}=\phi_{o}$. Now,

$$
u_{0}^{\prime}(t) \leq f\left(t, u_{0, t}\right)=F\left(t, u_{0, t}, u_{0, t}\right), \quad u_{0,0} \leq \phi_{o},
$$

and

$$
v_{0}^{\prime}(t) \geq f\left(t, v_{0, t}\right) \geq F\left(t, v_{0, t}, u_{0, t}\right), \quad v_{0,0} \geq \phi_{o},
$$

imply that $u_{0}(t)$ and $v_{0}(t)$ are lower and upper solutions of (3.21), respectively. Also,

$$
u_{0,0} \leq u_{1,0} \leq v_{0,0} .
$$

Thus, it follows from Theorem 2.4 that

$$
u_{0, t} \leq u_{1, t} \leq v_{0, t} \quad \text { for every } t \in J .
$$

Now, consider the following IVP for functional differential equation:

$$
u^{\prime}(t)=F\left(t, u(t), u_{1, t}\right), \quad u_{0}=\phi_{o}=u_{1,0}, \quad t \in J .
$$

Employing the earlier arguments, we find that (3.25) has a unique solution $u_{2}(t)$, with

$$
u_{2,0}=\phi_{o} .
$$

In view of the nondecreasing nature of $F(t, \phi, \psi)$, it follows that

$$
u_{1}^{\prime}(t)=F\left(t, u_{1, t}, u_{o, t}\right) \leq F\left(t, u_{1, t}, u_{1, t}\right), \quad u_{1,0}=\phi_{o},
$$

which implies that $u_{1}(t)$ is a lower solution of (3.25). Similarly, it can be shown that $v_{0}(t)$ is an upper solution of (3.25), and

$$
u_{1,0} \leq u_{2,0} \leq v_{0,0} .
$$

Hence, by Theorem 2.4, there exists a solution $u_{2, t}$ such that

$$
u_{1, t} \leq u_{2, t} \leq v_{0, t} \quad \text { for every } t \in J .
$$


Continuing in this way, we obtain a monotone sequence $\left\{u_{n, t}\right\}$ satisfying

$$
u_{0, t} \leq u_{1, t} \leq u_{2, t} \leq \ldots \leq u_{n, t} \leq v_{0, t} \quad \text { for } \quad t \in J
$$

where the element $u_{n, t}$ of the sequence is a solution of the IVP

$$
u^{\prime}(t)=F\left(t, u(t), u_{n-1, t}\right), \quad u_{0}=\phi_{o}=u_{n, 0} \quad, t \in J .
$$

Since the sequence $\left\{u_{n, t}\right\}$ is monotone, it follows that it has a pointwise limit $x_{t}$. To show that $x_{t}$ is in fact a solution of (2.1), we notice that $u_{n, t}$ is a solution of the following linear IVP for functional differential equation:

$$
\begin{aligned}
u^{\prime}(t) & =F\left(t, u_{n, t}, u_{n-1, t}\right), \quad u_{n, 0}=\phi_{o}, t \in J \\
& =\sigma_{n, t} .
\end{aligned}
$$

Since $F$ is a continuous function of $t$ on $[0, T]$, it follows that $\left\{\sigma_{n, t}\right\}$ is bounded on $[0, T]$. Also,

$$
\operatorname{Lim}_{n \rightarrow \infty} \sigma_{n, t}=F\left(t, x_{t}, x_{t}\right)=f\left(t, x_{t}\right), t \in J .
$$

Thus, from (3.26), we have

$$
u_{n, t}=\phi_{o}+\int_{0}^{t} \sigma_{n, s} d s .
$$

This proves that $\left\{u_{n, t}\right\}$ is uniformly bounded on $J$. Passing on to the limit $n \rightarrow \infty$, we obtain

$$
x_{t}=\int_{0}^{t} f\left(s, x_{t}\right) d s+\phi_{o}
$$

which is a solution of (2.1).

Now, we show that the convergence is of order $k \geq 2$. For that, we define

$$
e_{n}(t)=x(t)-u_{n}(t), a_{n}(t)=u_{n+1}(t)-u_{n}(t), t \in \bar{J},
$$

so that, $e_{n}(t) \geq 0, a_{n}(t) \geq 0$ and $e_{n}(s)=x(s)-u_{n}(s)=x_{0}-u_{n, 0}=\phi_{o}-\phi_{o}=0$, $a_{n}(s)=0, s \in[-\tau, 0]$.

In view of assumption $\left(A_{3}\right)$ and the generalized mean value theorem, we have

$$
\begin{aligned}
e_{n+1}^{\prime}(t)= & x^{\prime}(t)-u_{n+1}^{\prime}(t) \\
= & f\left(t, x_{t}\right)-F\left(t, u_{n+1, t}, u_{n, t}\right) \\
= & \sum_{i=0}^{k-1} \frac{\partial^{i}}{\partial \phi^{i}} f\left(t, u_{n, t}\right) \frac{\left(x_{t}-u_{n, t}\right)^{i}}{i !}+\frac{\partial^{k}}{\partial \phi^{k}} f\left(t, \xi_{t}\right) \frac{\left(x_{t}-u_{n, t}\right)^{k}}{k !} \\
& -\sum_{i=0}^{k-1} \frac{\partial^{i}}{\partial \phi^{i}} f\left(t, u_{n, t}\right) \frac{\left(u_{n+1, t}-u_{n, t}\right)^{i}}{i !}+m_{k}\left(u_{n+1, t}-u_{n, t}\right)^{k} \\
\leq & \sum_{i=1}^{k-1} \frac{\partial^{i}}{\partial \phi^{i}} f\left(t, u_{n, t}\right) \frac{1}{i !}\left(e_{n, t}^{i}-a_{n, t}^{i}\right)+\frac{M}{k !} e_{n, t}^{k}+m_{k} a_{n, t}^{k} \\
\leq & \sum_{i=1}^{k-1} \frac{\partial^{i}}{\partial \phi^{i}} f\left(t, u_{n, t}\right) \frac{1}{i !} \sum_{j=0}^{i-1} e_{n, t}^{i-1-j} a_{n, t}^{j}\left(e_{n, t}-a_{n, t}\right)+C e_{n, t}^{k} \\
= & \sum_{i=1}^{k-1} \frac{\partial^{i}}{\partial \phi^{i}} f\left(t, u_{n, t}\right) \frac{1}{i !} \sum_{j=0}^{i-1} e_{n, t}^{i-1-j} a_{n, t}^{j} e_{n+1, t}+C e_{n, t}^{k},
\end{aligned}
$$


where

$$
C=\frac{M+m_{k} k !}{k !}, e_{n+1, t}=e_{n, t}-a_{n, t}, \quad \frac{\partial^{k}}{\partial \phi^{k}} f\left(t, \xi_{t}\right) \leq M, \quad e_{n, t} \geq a_{n, t} .
$$

Taking

$$
Q_{n, t}=\sum_{i=1}^{k-1} \frac{\partial^{i}}{\partial \phi^{i}} f\left(t, u_{n, t}\right) \frac{1}{i !} \sum_{j=0}^{i-1} e_{n, t}^{i-1-j} a_{n, t}^{j},
$$

the expression (3.29) becomes

$$
e_{n+1}^{\prime}(t) \leq Q_{n, t} e_{n+1, t}+C e_{n, t}^{k} .
$$

Notice that

$$
\lim _{n \rightarrow \infty} Q_{n, t}=f_{\phi}\left(t, x_{t}\right) .
$$

This implies that $\left\{Q_{n, t}\right\}$ is bounded .It follows that there exists some $L>0$, such that $Q_{n, t} \leq L$. Thus, we have

$$
\begin{aligned}
e_{n+1}^{\prime}(t) & \leq L e_{n+1, t}+C e_{n, t}^{k}, e_{n+1,0}=0 \leq \phi_{o}, t \in J \\
& =w^{\prime}(t) \text { (say). }
\end{aligned}
$$

Clearly, $w^{\prime}(t) \geq 0$. Since $e_{n}(t) \leq w(t)$ and $w(t)$ is nondecreasing in $t$, we get

$$
w(t) \leq L_{2} \int_{0}^{t} e_{n-1, s}^{k} d s+L \tau \int_{0}^{t} w(s) d s .
$$

Noting that $w(0)=0$ and using Gronwall's inequality [8], (3.30) can be written as

$$
\begin{aligned}
e_{n}(t) & \leq w(t) \leq L_{2} \int_{0}^{t} e^{L \tau(t-S)} e_{n-1, s}^{k} d s \\
& \leq L_{2} \frac{e^{L \tau T}}{L \tau} \max e_{n-1, t}^{k} \quad \text { where } t \in J .
\end{aligned}
$$

Consequently,

$$
\left\|e_{n}(t)\right\| \leq C\left\|e_{n-1, t}\right\|^{k}
$$

where $C=L_{2} \frac{e^{L \tau T}}{L \tau}$. This completes the proof.

\section{References}

[1] Bellman, R. and Kalaba, R., Quasilinearization and Nonlinear Boundary Value Problems, American Elsevier, New York 1965.

[2] Lakshmikantham, V., An extension of the method of quasilinearization, J. Optim. Theory Appl. 82(1994), 315-321.

[3] Lakshmikantham, V., Further improvement of generalized quasilinearization, Nonlinear Analysis 27 (1996), 315-321.

[4] Lakshmikantham, V. and Leela, S., Differential and Integral Inequalities Vol. I \& II, Academic Press 1969. 
[5] Lakshmikantham, V., Leela, S., and McRae, F.A., Improved generalized quasilinearization method, Nonlinear Analysis 24 (1995), 1627-1637.

[6] Lakshmikantham, V. and Shahzad, N., Further generalization of generalized quasilinearization method, J. Appl. Math. Stoch. Anal. 7 (1994), 545-552.

[7] Lakshmikantham, V., Shahzad, N., and Nieto, J.J., Method of generalized quasilinearization for periodic boundary value problems, Nonlinear Analysis 27 (1996), $143-151$.

[8] Lakshmikantham, V. and Vatsala, A.S., Generalized Quasilinearization for Nonlinear Problems, Kluwer Academic Publishers, Boston 1998.

[9] Nieto, J.J., Generalized quasilinearization method for a second order ordinary differential equation with Dirichlet boundary conditions, Proc. Amer. Math. Soc. 125 (1997), 2599-2604.

[10] Shahzad, N. and Sivasundaram, S., Extended quasilinearization method for boundary value problems, Nonlinear World 2 (1995), 311-319.

[11] Shahzad, N. and Vatsala, A.S., An extension of the Method of generalized quasilinearization for second order boundary value problems, Applicable Analysis $\mathbf{5 8}$ (1995), 77-83.

[12] Shahzad, N. and Vatsala, A.S., Improved generalized quasilinearization for second order boundary value problems, Dynam. Systems Appl. 4 (1995), 79-85. 


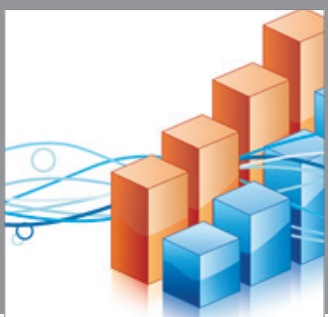

Advances in

Operations Research

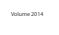

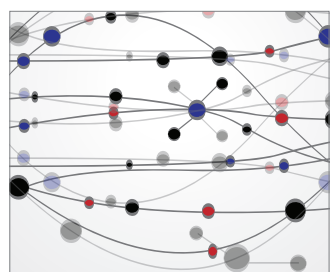

\section{The Scientific} World Journal
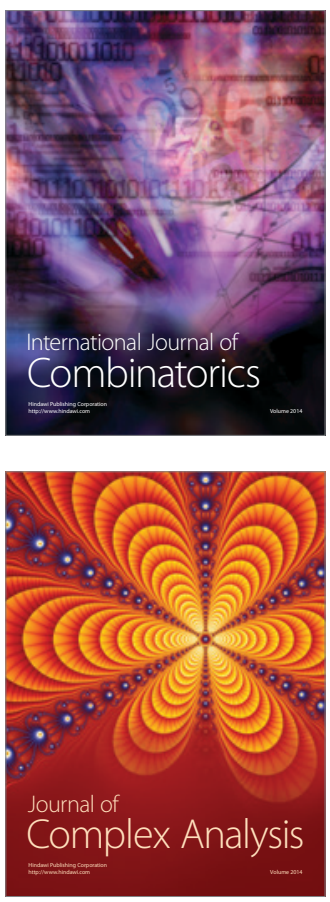

International Journal of

Mathematics and

Mathematical

Sciences
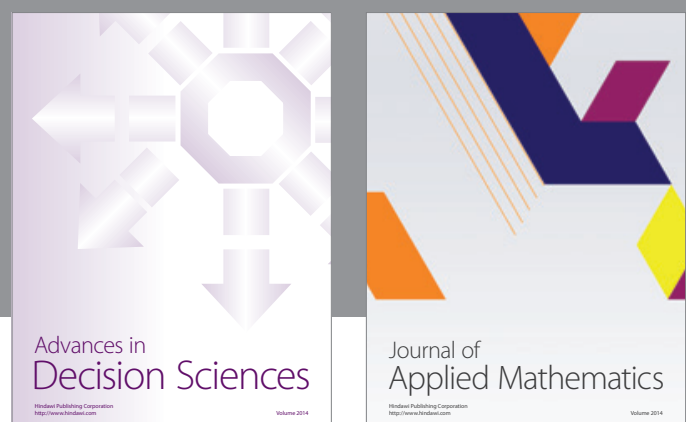

Journal of

Applied Mathematics
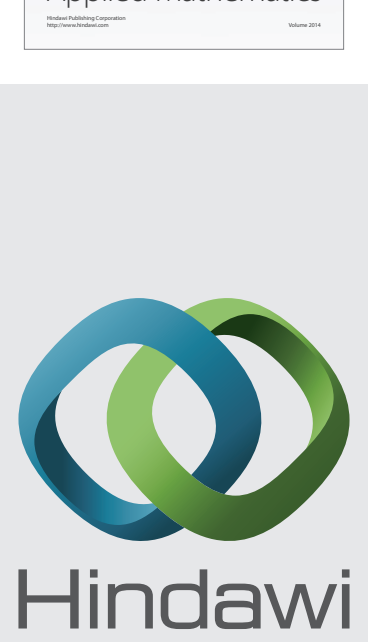

Submit your manuscripts at http://www.hindawi.com
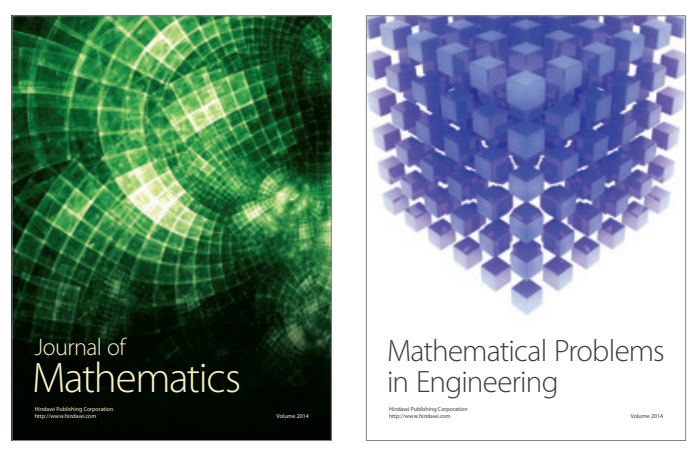

Mathematical Problems in Engineering
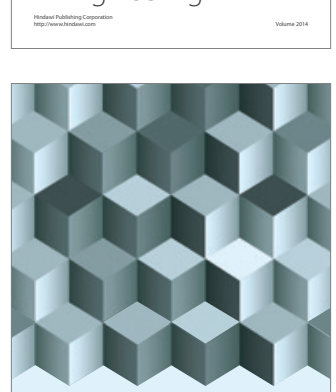

Journal of

Function Spaces
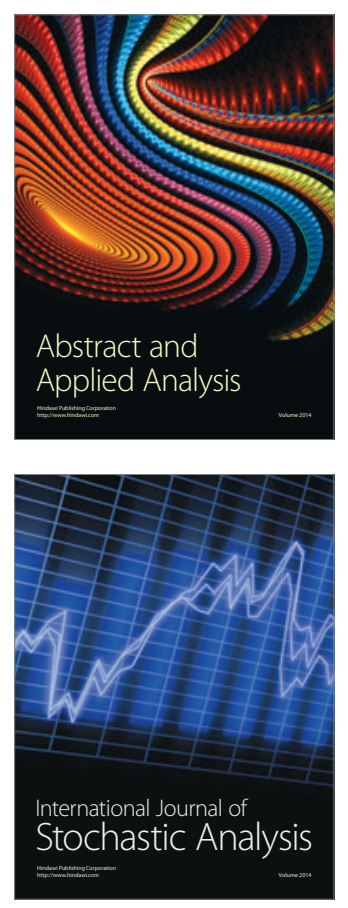

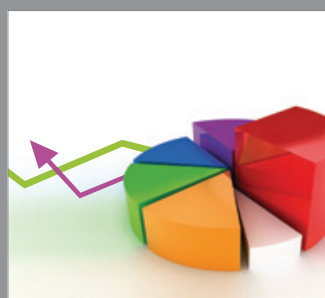

ournal of

Probability and Statistics

Promensencen
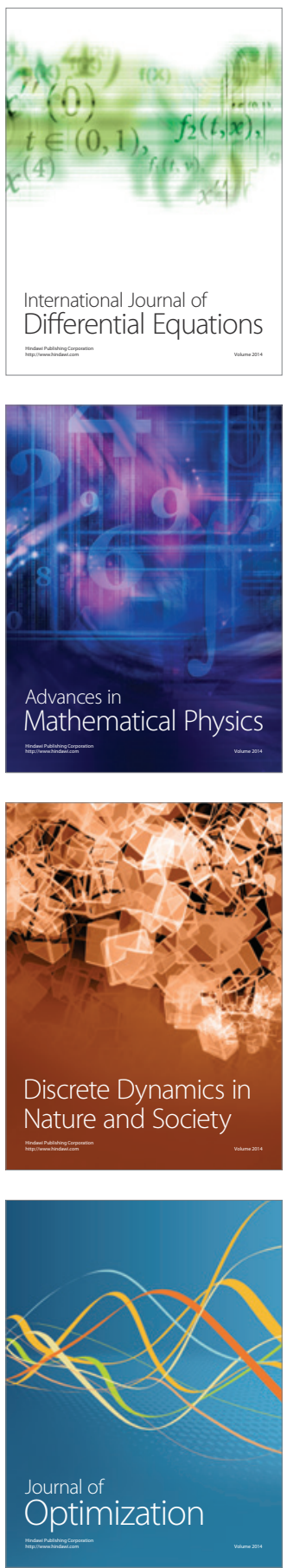